\title{
Environment, facilities, and management of hospital pens in growing and finishing pig farms: a descriptive study
}

\author{
Carlos Rodolfo Pierozan ${ }^{1 *}$, Cleandro Pazinato Dias², Caio Abércio da Silva ${ }^{3}$
}

\author{
1 Universidade Estadual de Londrina, Programa de Pós-graduação em Ciência Animal, Londrina, PR, Brazil. \\ ${ }^{2}$ Akei Animal Research, Fartura, SP, Brazil. \\ ${ }^{3}$ Universidade Estadual de Londrina, Departamento de Zootecnia, Londrina, PR, Brazil.
}

\begin{abstract}
The objective of this study was to describe the housing system in the hospital pens on growing and finishing (GF) pig farms. This work was developed by using a questionnaire and was conducted between January and April, 2016. Forty-four GF commercial farms (a total of 41,111 animals) were involved, seeking to raise issues concerning the facilities of the hospital pens, environment, management, feeding and the reasons for transferring the animals to these accommodations. About $98 \%$ of the evaluated farms had, at least, one hospital pen. Most of these farms presented a percentage of animals evaluated (PAE) in the infirmary, of less than $1.5 \%$ of the total capacity of the animal housing on the farm. In $93.48 \%$ of the farms, the criterion used to transfer the animals to the infirmaries was when they were identified as "impaired, hurt, suffering". In $47.83 \%$ of the cases, there were no differences between the hospital and common pens regarding the general management of the animals. The facilities and environment characteristics were similar in almost all hospital pens. Respiratory problems were the main reason for transferring pigs from the common to hospital pens. Under the conditions of this study, sufficient hospital pens exist on farms and overcrowding or competition for resources among pigs are avoided. However, there is no established standard among respondents about where to place the recovered animals.
\end{abstract}

Key Words: animal welfare, compromised pig, infirmary pen, recovery, sick

\section{Introduction}

Hospital pens, or infirmary pens, are often used to facilitate the treatment of sick animals and separate them from those that are healthy (Fraser et al., 2013). In these pens, pigs are able to recover without competing with healthy animals for water, food, or rest areas (Pineiro et al., 2014).

In Denmark, there is a law that specifies how a hospital pen should be designed, which addresses requirements, such as bedding material, cooling, heating, and extra space in these facilities (Thomsen et al., 2016). The correct use of an infirmary pen is relevant and strongly associated with the welfare of animals. Consequently, there are many technical recommendations about the care that must be provided to animals in these units (Charbonneau, 2005; Morés, 2007; Holyoake et al., 2012). In Brazil, it is recommended that

Received: January 5, 2017

Accepted: August 11, 2017

*Corresponding author: carlospierozan@hotmail.com

http://dx.doi.org/10.1590/S1806-92902017001100001

How to cite: Pierozan, C. R.; Dias, C. P. and Silva, C. A. 2017. Environment, facilities, and management of hospital pens in growing and finishing pig farms: a descriptive study. Revista Brasileira de Zootecnia 46(11):831-838.

Copyright (c) 2017 Sociedade Brasileira de Zootecnia. This is an Open Access article distributed under the terms of the Creative Commons Attribution License (http://creativecommons.org/licenses/by/4.0/), which permits unrestricted use, distribution, and reproduction in any medium, provided the original work is properly cited. hospital pens be distant or attached to the barn of healthy animals, but with an independent circulation area (Morés, 2006). However, in Brazilian pig farms that only develop the growing and finishing (GF) phases, hospital pens are routinely observed inside the barn. Furthermore, there is no consistent information about how sick pigs are being housed and managed in practice.

Temple et al. (2012) evaluated the presence of hospital pens in different production systems for GF pigs in France and Spain, but the particularities of these accommodations were not described, reflecting the limited information available about the management characteristics of ailing animals and the structure of hospital pens on GF pig farms. Thus, identifying these characteristics in a region with a tradition of pig farming could represent a valuable profile of the conditions under which these animals are kept. Such an evaluation will also provide greater insight into how the unwell pigs are treated and possible ways to improve their recovery conditions. The information provides a basis for future research to seek a more advanced understanding of specific situations of sick animals on farms, enabling faster and objective solutions.

In this context, two questions were proposed to enhance the discussion on this subject: Is there a standard for facilities and management of animals in hospital pens on farms that adopt the intensive production system? Are the 
facilities and management offered in these pens suitable for the recovery of animals? Thus, this study aimed to describe the housing system in hospital pens on GF pig farms, identifying their strengths and weaknesses.

\section{Material and Methods}

The project was approved by the Ethical Committee on Animal Use (CEUA) (Circular Letter CEUA no. 86/2016).

Data were collected between January and April of 2016 and included 47 batches of pigs housed in 47 commercial GF farms (one batch per farm), integrated to four cooperatives $(\mathrm{A}=12$ farms; $\mathrm{B}=12$ farms; $\mathrm{C}=12$ farms; and $\mathrm{D}=11$ farms). The farms totaled a herd of 41,111 animals of commercial genetics. The batch was defined as the group of piglets that left the nursery phase and was housed and maintained on the GF farm to slaughter. All batches were managed in an all-in/all-out production system. On each farm, the data were collected on a single day and by a single evaluator between the morning and afternoon. During the period of data collection, the minimum and maximum temperatures measured within each farm were $25.5 \pm 2.5^{\circ} \mathrm{C}$ (range from $21.0-32.0{ }^{\circ} \mathrm{C}$ ) and $30.4 \pm 3.1{ }^{\circ} \mathrm{C}$ (range from $25.0-35.0^{\circ} \mathrm{C}$ ), respectively, and the relative humidity was $65.5 \pm 10.3 \%$ (range from $47.0-81.0 \%$ ).

The selection of the farms was based on the historical performance of four to six previous batches (high and low performance), as well as through technical suggestions of each cooperative. The first stage of the study was to develop a standard questionnaire to collect the information, which was based on the recording sheets of the pen facilities for growing pigs of the Welfare Quality ${ }^{\circledR}$ project - assessment protocol for pigs (Welfare Quality, 2009), with some adjustments. This questionnaire was supplemented with items established by the authors with open, semi-open, and closed questions (Table 1). Before data collection, a pre-test was carried out. In this test, the questionnaire was applied to a farm and included, excluded, or modified the questions according to the understanding of the caretaker and of the suggestions made by the authors of the study.

The face-to-face questionnaire was primarily answered by the farm owner and sometimes by employees or the caretaker responsible for animal care. In general, the questions were related to the facilities and environment of the hospital pens, the management practices, and feeding provided, criteria on which the farm manager based the transfer of animals to the hospital pens, reasons that the animals were transferred, as well as the destination of the animals after recovery.

The data collected from each farm were transferred to a spreadsheet developed in Microsoft Excel (Microsoft
Office Professional Plus 2010) and the function "FILTER" was used for initial data observations and to facilitate the identification of any errors in the entered values. The variables were divided into quantitative (discrete and continuous) (Table 2 and Figure 1) and qualitative, regarding the management of the animals (Table 3) and the facilities and pen environment (Table 4). The reasons the animals were in the hospital pens were also addressed (Table 5).

For the calculation of the quantitative variables, the following criteria were defined. The usable space $\left(\mathrm{m}^{2} / \mathrm{pig}\right)$ was calculated by subtracting from the total area of the hospital pens used on the farm, the area occupied by feeders, shallow pools, and other structures and then dividing this value by the number of animals in these pens. For pens with an ill-defined shallow pool area (mini shallow pool), which varied greatly in size due to the water waste or working state of the dripping system, the area of the mini shallow pool was not subtracted in calculating the usable space. The percentage of animals evaluated in hospital pens in relation to all animals on the farm (PAE) was calculated by the sum of the animals in all hospital pens on the farm, divided by the total number of animals on the farm, then multiplied by 100. In calculating the percentage of supported animals in hospital pens regarding all animals on the farm (PAS), the number of animals supported by these pens was considered: sum of the total usable space of all hospital pens on the farm $\left(\mathrm{m}^{2}\right)$ divided by $1.15 \mathrm{~m}^{2}\left(0.01 \mathrm{~m}^{2}\right.$ was considered the space required for each kilogram of animal, based on an average estimate of $115 \mathrm{~kg}$ of final live weight) (Machado, 2014). When the number of animals supported in hospital pens showed decimal values, it was always rounded down. This value was subsequently divided by the total number of animals on the farm, then multiplied by one hundred, resulting in the PAS. The total number of animals in

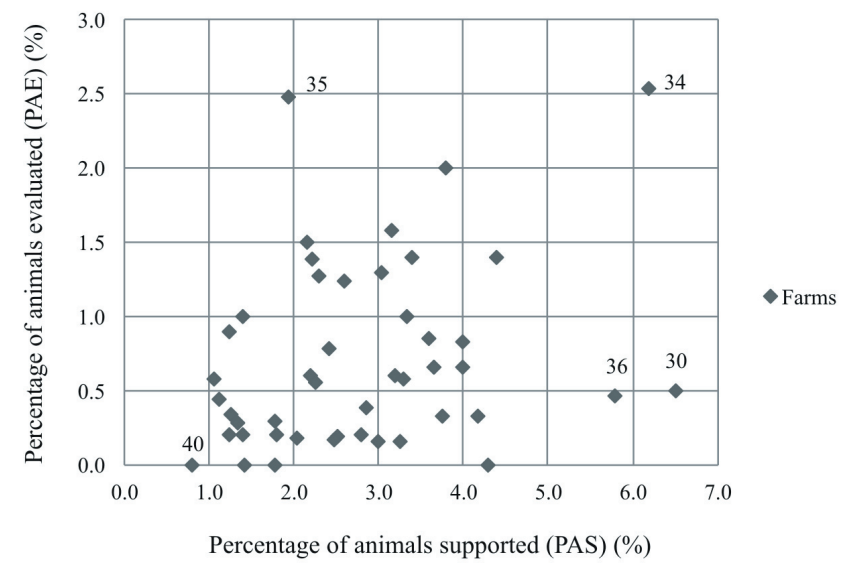

Figure 1 - Distribution of the 46 farms for PAE and PAS. 
hospital pens on the farm was divided by the sum of the areas of the eating places in the feeders, resulting in the variable "animals per feeder space"; correspondingly, it was also divided by the sum of drinking places, resulting in the variable "animals per drinker". For these calculations, only the clean and functional feeders and drinkers were considered. The temperature $\left({ }^{\circ} \mathrm{C}\right)$ inside the pens was measured with a digital thermometer, placed in the center of each hospital pen, where there was at least one animal. For qualitative variables, the answers received from open questions were transformed into categories according to the similarity of the answers. About the answers to the variable "mode/time for transfer", some respondents reported the use of some type of cart to transport the animals with limited mobility to the hospital pen. Consequently, the variable "cart for animals with limited mobility" was created.
In the exploratory analysis, measures of central tendency (mean and median) and dispersion (standard deviation, quartiles, and amplitude) were calculated for the quantitative variables, while frequencies and percentages of occurrence within each category were obtained for the qualitative variables, using the "Rcmdr" package of R software ( $\mathrm{R}$ Core Team, R version 3.3.1, Vienna, Austria, 2016). The "farm" was considered the experimental unit for all measurements in the exploratory analysis. Later, it was found that it would be more significant that some qualitative variables (Table 4) were analyzed at the "pen" level because these are regarded as characteristics inherent to each pen (with differences among pens within the same farm), and the qualitative variable "reasons why the animals were in hospital pens" analyzed at the "animal" level because it is self-intrinsic to the animals.

Table 1 - Main items of the questionnaire used to collect data in 46 evaluated farms

\begin{tabular}{|c|c|}
\hline Item & Description/answer option \\
\hline Farm $^{1}$ & Name and order number of the evaluated farm \\
\hline Respondent $^{1}$ & Name and function (owner, manager, caretaker, other) of the respondent \\
\hline Presence of hospital pens ${ }^{2}$ & 1. Yes; 2. No \\
\hline Number of hospital pens ${ }^{2}$ & How many hospital pens are there in each room/barn? \\
\hline Time for transfer/way ${ }^{2}$ & What are the time and the way you transfer the animals? \\
\hline Feeding $^{2}$ & Is there any difference in the diet of the animals in the hospital pens? What? \\
\hline Feeding mode ${ }^{2}$ & What is the feeding frequency of the animals? \\
\hline General management ${ }^{2}$ & How is the general management of hospital pens relative to common pens? \\
\hline Pen/room $/$ barn $^{3}$ & Number of the evaluated hospital pen and in which room/barn it was located. \\
\hline Pigs in the pen ${ }^{3}$ & Total number of pigs housed in the pen. \\
\hline Pen size ${ }^{3}$ & Length; width \\
\hline Temperature $\left({ }^{\circ} \mathrm{C}\right)^{3}$ & Measured in the center of each hospital pen, at the height of the animals. \\
\hline Feeder $^{3}$ & $\begin{array}{l}\text { Type (1. Circular; 2. Horizontal; 3. Longitudinal/linear dump; 4. On the floor } \mid 1 \text {. Dry feed; } 2 \text {. Wet feed } \mid \text { 1. One feeder } \\
\text { space; 2. Two or more feeder spaces); clean (1. Yes; 2. No); functional (1. Yes; } 2 \text {. No); length; width }\end{array}$ \\
\hline Drinker $^{3}$ & Type (1. Nipple; 2. Bowl); clean (1. Yes; 2. No); functional (1. Yes; 2. No) \\
\hline Shelter inside the pen ${ }^{3}$ & 1. Yes; 2. No \\
\hline Access to the outside ${ }^{3}$ & 1. Yes; 2. No \\
\hline
\end{tabular}




\section{Results}

Of the 47 farms, $46(97.87 \%)$ had at least one hospital pen (Table 2). Thus, a total of 46 farms were assessed and included 150 hospital pens. In 42 farms $(91.30 \%)$ and 97 pens $(64.67 \%)$, at least one animal was housed. The evaluated farms totaled 41,111 housed animals, of which 299 (about $0.73 \%$ ) were in hospital pens. On all farms with one or more hospital pens, these pens were located in the same barn where healthy animals were housed.

On most farms, the PAS was $<4.5 \%$ and the PAE was $<1.5 \%$ (Figure 1). A single farm (no. 40$)(2.17 \%$ ) had a PAS $<1.0 \%$, while in three farms (no. 30,34 , and 36 ) (6.52\%), the PAS was $>5.0 \%$. One farm (no. 35$)(2.17 \%$ ) presented PAE higher than the PAS.

As a criterion used to transfer the animals to the hospital pens, the people responsible for most of the farms reported transferring the animals when it was observed that the animal was impaired, hurt, or suffering (Table 3). Regarding the mode and time of the transfer, most reported that they did it immediately, either with or without the assistance of other people and/or management tools. Once the previous question was presented as an open question, $26.09 \%$ of these respondents also reported using some type of cart to transport the animals with limited mobility to the hospital pens. Regarding the destination of the recovered

Table 2 - Descriptive values of quantitative variables related to facilities and environment of the hospital pens

\begin{tabular}{|c|c|c|c|c|c|c|c|c|}
\hline Variable & Farm (n) & Mean & $\mathrm{SD}$ & Low & Lower quartile & Median & Upper quartile & Upper \\
\hline Total number of hospital pens & 47 & 3.2 & 1.8 & 0.0 & 2.0 & 3.0 & 4.0 & 9.0 \\
\hline Usable space $\left(\mathrm{m}^{2} / \mathrm{pig}\right)$ & 42 & 4.1 & 2.7 & 1.0 & 2.1 & 3.8 & 5.0 & 13.8 \\
\hline Animals per feeder space & 36 & 1.5 & 1.3 & 0.2 & 0.5 & 1.3 & 2.0 & 4.8 \\
\hline Animals per drinker & 39 & 2.5 & 2.3 & 0.5 & 1.0 & 2.0 & 3.2 & 13.5 \\
\hline Temperature inside the pens $\left({ }^{\circ} \mathrm{C}\right)$ & 42 & 29.6 & 3.4 & 24.1 & 27.0 & 30.1 & 32.3 & 35.7 \\
\hline
\end{tabular}

SD - standard deviation.

Table 3 - Frequency and percentage of occurrence, in each category, of variables related to management in hospital pens in 46 evaluated farms

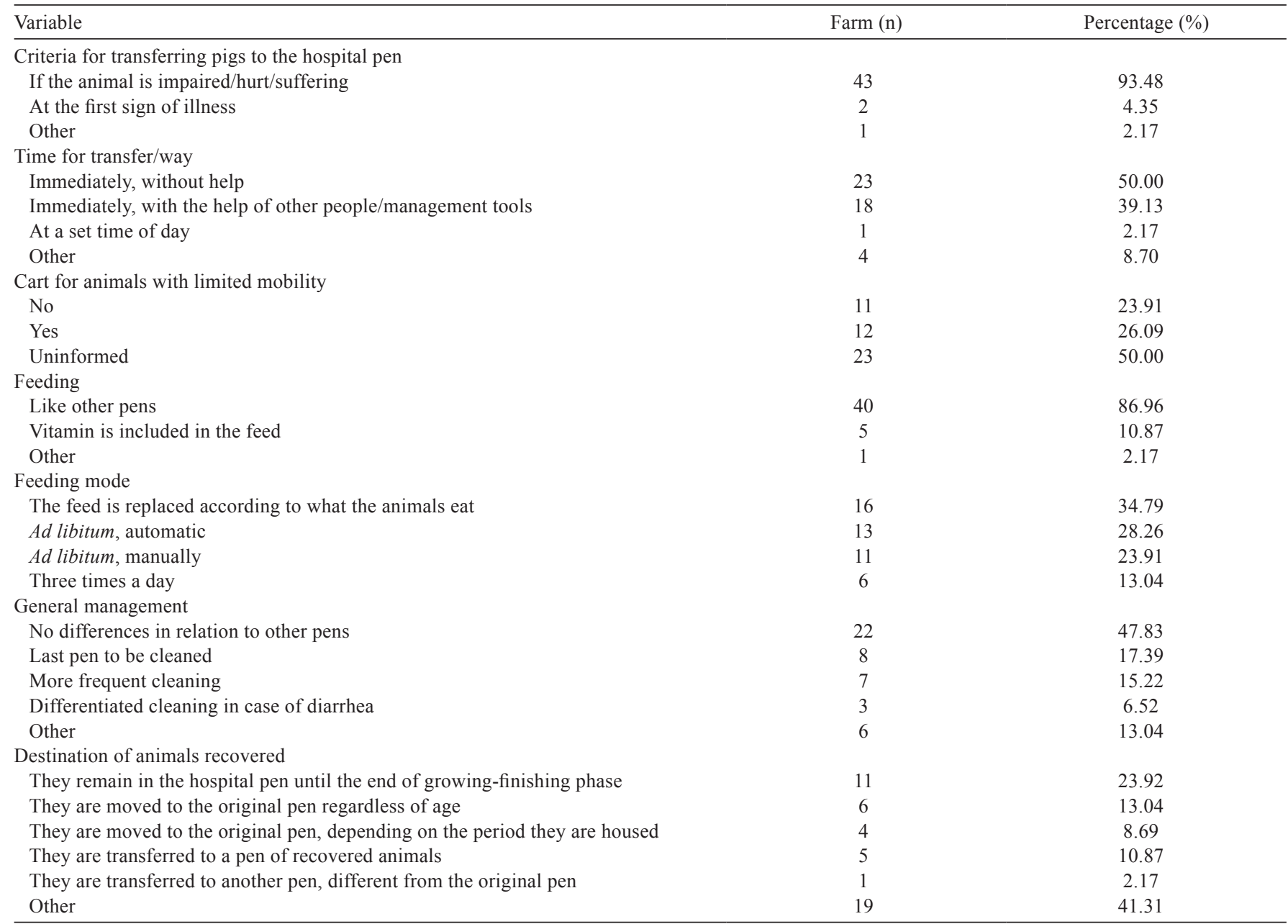


animals, most respondents reported keeping these animals in hospital pens until the end of the GF phases. Others reported relocating the recovered pigs to the original pen or transferring them to another pen distinct from the original, or to a recovery pen; furthermore, $41.31 \%$ answered that it depended on the various conditions, for example, time of housing, availability of empty pens, and the capacity of the hospital pens.

Generally, the pens had similar characteristics, without shelter or a heating source for the animals and without external access (Table 4). Few of them had some form of enrichment. Most, however, had a fully solid floor (no slats) and a shallow pool (mini or entire shallow pool). The cleaning of the pens ranged mainly between very good, good, and regular. However, there were nine pens classified as having a poor cleaning.

Given that some farms had many animals housed in hospital pens, no inquiries were made as for the reasons for the transfer because it was not possible to accurately identify the original motivation. Therefore, of the 299 animals evaluated, $46.2 \%$ did not have a reason for housing animals in the described hospital pens (Table 5). Considering other factors, respiratory problems were reported as the main reason for the transfer of animals to the hospital pen, followed by locomotor problems, rectal prolapse, and encephalitis. Heart attack, fights, tail biting, and diarrhea were the least frequently reported problems.

Table 4 - Frequency and percentage of occurrence, in each category, of variables related to facilities and environment in 97 evaluated hospital pens

\begin{tabular}{lcc}
\hline Variable & Pen (n) & Percentage (\%) \\
\hline $\begin{array}{l}\text { Shelter } \\
\quad \text { No }\end{array}$ & 97 & 100.00 \\
Heating & 97 & 100.00 \\
$\quad$ No & & \\
Access to the outside & 1 & 1.03 \\
$\quad$ Yes & 96 & 98.97 \\
$\quad$ No & & \\
Enrichment & 15 & 15.46 \\
Yes & 82 & 84.54 \\
$\quad$ No & & \\
Floor surface & 10 & 10.30 \\
$\quad$ Partly slatted & 87 & 89.70 \\
Solid & & \\
Type of shallow pools in the pens & 11 & 11.34 \\
$\quad$ Entire shallow pool & 70 & 72.16 \\
Mini-shallow pool & 16 & 16.50 \\
$\quad$ Without shallow pool & & \\
Pen cleaning & 9 & 9.28 \\
Poor & 25 & 25.77 \\
Regular & 37 & 38.15 \\
Good & 26 & 26.80 \\
Very good & & \\
\hline
\end{tabular}

\section{Discussion}

The percentage of assessed farms that had at least one hospital pen was higher than that reported by Temple et al. (2012) in the straw-bedded system (43\% had a hospital pen), conventional system (70\%; "white" pigs housed on concrete floors), and intensive system for Iberian pigs (90\%) on farms located in France and Spain. This emphasizes the concern to provide a separate place for sick animals in the intensive system of Brazilian pig production, an assertion reinforced by finding an average of about three hospital pens per farm (Table 2).

The transfer of animals to a less competitive pen can facilitate feed intake and improve recovery and animal welfare (Fogsgaard et al., 2016). In normal pens, up to 10 pigs per feeder space (Zulovich, 2012), 8-10 per nipple drinker, and up to 18 per bowl drinker (Brustolini, 2014) are recommended. Averages of less than two and three animals were found for the variables "animals per feeder space" and "animal per drinker", respectively, which may indicate that on most of the evaluated farms, there are few disputes among pigs for food and water.

The estimated thermal comfort zone ranges from $15-24{ }^{\circ} \mathrm{C}$ for growing pigs and from $14-21{ }^{\circ} \mathrm{C}$ for finishing pigs (Gonyou et al., 2006). However, the thermostatic setting changes when the animals display a febrile response, such that at increased temperatures, the animal feels colder than it would under normal circumstances (Hart, 1988). In the present study, the average temperature recorded in the pens was around 6 and $9{ }^{\circ} \mathrm{C}$ beyond the upper limit for pigs in growing and finishing phases, respectively. These values may perhaps be considered higher than necessary, even for sick animals that develop a febrile response.

In GF units that practice downtime between batches, the hospital room should provide housing for $6 \%$ of the pigs in installation (considering smaller pens for sick animals

Table 5 - Problems of the animals $(n=299)$ in hospital pens

\begin{tabular}{lcc}
\hline Reason & Animal affected (n) & Percentage (\%) \\
\hline Heart attack & 1 & 0.3 \\
Fight (active) & 1 & 0.3 \\
Fight (victim) & 1 & 0.3 \\
Tail biting (active) & 4 & 1.3 \\
Tail biting (victim) & 6 & 2.0 \\
Diarrhea & 6 & 2.0 \\
Encephalitis & 13 & 4.4 \\
Rectal prolapse & 13 & 4.4 \\
Locomotor problem & 20 & 6.7 \\
Respiratory problem & 34 & 11.4 \\
Other & 29 & 9.7 \\
Recovered & 33 & 11.0 \\
Uninformed & 138 & 46.2 \\
\hline
\end{tabular}


and larger pens for recovered animals) and offer $1.5 \mathrm{~m}^{2} / \mathrm{pig}$ (Morés, 2007). In the present study, the pens of recovered animals were not evaluated and $1.15 \mathrm{~m}^{2} / \mathrm{pig}$ was considered in the PAS calculation (as described in the Material and Methods section). In this matter, it was observed that the majority of the farms had a PAE $<1.5 \%$, allowing to hypothesize that a PAS around $2 \%$ for the animals effectively sick would be sufficient. This proposition gains consistency when considering that the average usable space per animal was large (Table 2) and only one farm exceeded the PAS limit. It was also observed that almost all respondents reported transferring the animal to the hospital pen when they realized that the pig was impaired, hurt, or suffering, rather than when observing the first signs of illness. If this transfer is performed at the appropriate time, the previous consideration about PAS is valid because the PAE is real. However, if the transfer had been late, the PAE would have been underestimated and, in this sense, an assumption that $2 \%$ PAS would be sufficient does not hold.

Also, considering the criteria used for transferring animals to the hospital pens, Thomsen et al. (2016) questioned 508 Danish producers on the reasons they had not transferred sick pigs to the hospital pens and found that $77 \%$ reported that "it was not necessary to do it" because the animal was not "sick enough". The authors cited that producers may have a different threshold than the Danish regulatory authorities to define a pig as "sick enough" to warrant special care. Similarly, Mullan et al. (2009), assessing six commercial GF farms, found that $1.39 \%$ of all pigs housed in common pens (not hospital pens) required hospitalization. The authors attributed this to either the animals were "lost" (forgotten by mistake in the common pen) by the producers or that the producers had a different threshold to consider a pig as requiring hospitalization. It is probable that this latter phenomenon also occurs among Brazilian producers. Indeed, in support of this hypothesis, in Brazil, there is no standardized guideline about when farmers should place unwell animals in a hospital pen. Suspected signs of disease can be misleading and a successful detection rate can be improved using more effective criteria and training (Weary et al., 2009).

Regarding the form and timing of the transfer, the management was considered appropriate because most respondents reported transferring the animals immediately. However, as already debated, the criteria used in deciding when an animal should be transferred from the common pen to a hospital pen remains a major unresolved issue. Further studies should better evaluate these criteria in an attempt to establish a standard threshold to ensure that transfer of an ailing animal occurs at the appropriate time.
Regardless of the state of the pig, it should never be lifted by the ears, head, tail, or legs and should not be dragged or moved by brutal force (Ludtke et al., 2010). However, for animals with locomotion difficulty, it was uncertain as to how they were transported to the hospital pens on the assessed farms that lacked a cart or other appropriate equipment. This tool could facilitate the work of the caretaker, as well as improve the welfare of pigs, being relatively easy to implement.

Munsterhjelm et al. (2015) observed a 13-99\% reduction in feed intake for growing pigs (affected by lameness or tail biting), depending on the type of disorder, degree of possible recovery, and age of the animal. This shows that sick or injured pigs suffer from some degree of anorexia, requiring diets that promote ingestion and enable recovery. Few farms in this study offered a different diet to the animals housed in the hospital compared with common pens. Moreover, on some farms, the use of a restricted feeding system (feed is reset according to the intake of animals or offered several times a day) for animals in hospital pens was reported. In a restricted feeding system, we must consider that it is possible that a lack of feed or an amount of feed offered above that which the animals can consume immediately remains for extended periods in the feeder, deteriorating and becoming unpleasant to consume. Conversely, this feeding system allows a better monitoring of food intake compared with the ad libitum method.

Fomites, such as contaminated boots and coveralls, have been considered risk factors for pathogen transmission in pigs (Otake et al., 2002). Thus, cleaning the common pens, in particular, but also the hospital pens (where there is a greater probability of the existence of animals with infectious diseases), can prevent the transmission of pathogens to healthy animals. However, in this study, there were few farms where this type of care was reported to occur.

Most respondents reported to allow sick animals to recover in hospital pens until the end of the GF period. This could cause a high animal density until the end of the fattening phase. However, as discussed above, the PAE surpassed the PAS on only one farm. Hence, overcrowding of hospital pens was not an issue. Some recommendations indicate that recovered animals should be returned to the general population, if there is no evidence of potential impairment of welfare (Holyoake et al., 2012) or, preferably, be housed in large groups when fully recovered (Charbonneau, 2005). Yet, other guidelines include transferring recovered animals to recovery pens indefinitely (Morés, 2007). Presently, many respondents 
reported returning the animals to the original pen. This can lead to problems regarding animal welfare, particularly if the reintroduced animal is not recognized by other pigs in the pen. Aggressive behavior can be easily observed when unknown pigs are mixed with a new group (Stukenborg et al., 2011; Rhim et al., 2015). The aggression that results from the mixing of animals causes economic consequences and serious health issues. Most researchers concluded that this practice should be avoided (Erhard et al., 1997). On this theme, most respondents reported diverse answers, characterizing the lack of a standard management in this regard.

In cold regions, the hospital pen should provide additional heating (Morés, 2007). Moreover, one-third of the pen should be covered, aiming at creating a warm and comfortable zone, free from air currents (Charbonneau, 2005). None of the assessed pens offered shelter (coverage over part of the pen) or supplementary heating and this was not due to high temperatures during the evaluation period. Generally, a shelter is a fixed structure, which was not observed in any pen. Furthermore, no respondent mentioned providing heating at any time throughout the year, indicating that these basic essentials are not offered at any time, regardless of the weather.

There are no regulations or recommendations on the use of manipulation materials in an intensive rearing environment in Brazil. Chains and tires were the main materials found in the pens of farms with enrichment. Van de Weerd and Day (2009) mentioned that the use of chains and car tires as devices to enhance the welfare of animals is widespread on farms. Nonetheless, these objects are not recommended for long-term use and can rapidly lose their "novelty factor" (the animals quickly lose interest). The lack of enrichment materials found may be associated with cultural issues or the absence of Brazilian regulations concerning animal welfare, mainly in the production sector within the farms.

In the hospital pens of the assessed farms, the prevalence of solid floors can be considered a positive feature, although this type of flooring requires proper management of the shallow pool and more effective and frequent cleaning in relation to the pens with a slatted floor. For the welfare and recovery of compromised dairy cows, it is important that they can express their motivation to lie down in the hospital pen. In these instances, the type of flooring can be of higher priority than the original environment in which they live (Fogsgaard et al., 2016). No published studies were found about the motivation of pigs to lie down in clean areas. Sick pigs generally rest for extended periods, probably to conserve energy and to mount an immune response (Hart, 1988). Thus, it is possible that ailing pigs are motivated to lie down in clean and dry areas, increasing their resting time and reducing heat lost to the environment, reserving energy to combat the illness.

Regarding the reported reasons for transferring pigs to the hospital pens, it was expected that respiratory problems were the most reported because in the intensive production system, with batches of animals confined to a relatively small area, the transmission of infectious agents is favored. It was also found that animal victims of tail biting represented one of the least reported problems. The ratio of tail biting victims was slightly above the values observed by Temple et al. (2011) (0.9\% prevalence) and Temple et al. (2013) (0.7 and $0.4 \%$ prevalence in two consecutive evaluations), assessing, respectively, normal pens on 30 and 15 GF pig farms in Spain. According to Temple et al. (2013), if rare problems are considered (tail biting, for example), the evaluation of animals in hospital pens may be more representative than a random sample evaluation on the farm. Therefore, it is possible to assume that if there are animals on the farm with less frequent problems (such as tail biting or rectal prolapse) and the hospital pens are being used correctly, these animals will be housed in these pens.

The results of this study reflect the conditions of some farms in a traditional Brazilian pig farming region. Further studies are needed to investigate the conditions under which compromised pigs are maintained and treated in other regions. Such information will allow future comparisons and additional studies with hypotheses that explore the living conditions of these animals more deeply.

\section{Conclusions}

Under the conditions of this study, the farms have hospital pens in sufficient quantities to meet the demand, reflecting the absence of overcrowding and competition for resources among animals. The respondents in this study have their own criteria to define the destination of housing for the recovered animals; there is no standard in this regard. The facilities of the hospital pens and the feeding and management offered to animals within these accommodations on the evaluated farms are not differentiated from common pens.

\section{Acknowledgments}

The authors wish to thank the participating cooperatives and owners of the farms that allowed us to collect the data. We also thank the respondents for the valuable information provided. This work was conducted during a 
master's scholarship supported by the Conselho Nacional de Desenvolvimento Científico e Tecnológico (CNPq) to the first author.

\section{References}

Brustolini, A. P. L. 2014. Manejo alimentar e sistemas de alimentação na fase de terminação. p.668-676. In: Produção de suínos: Teoria e prática. Ferreira, A. H.; Carraro, B.; Dallanora, D.; Machado, G.; Machado, I. P.; Pinheiro, R. and Rohr, S. (Coord.). ABCS, Brasília.

Charbonneau, G. 2005. Effective treatment and handling of poor doing pigs. p.157-166. In: Proceedings of the 5th London Swine Conference. Ontario, Canada.

Erhard, H. W.; Mendl, M. and Ashley, D. D. 1997. Individual aggressiveness of pigs can be measured and used to reduce aggression after mixing. Applied Animal Behaviour Science 54:137-151.

Fogsgaard, K. K.; Herskin, M. S.; Gorden, P. J.; Timms, L. L.; Shearer, J. K. and Millman, S. T. 2016. Management and design of hospital pens relative to behavior of the compromised dairy cow: A questionnaire survey of Iowa dairy farms. Applied Animal Behaviour Science 175:50-55.

Fraser, D.; Duncan, I. J. H.; Edwards, S. A.; Grandin, T.; Gregory, N. G.; Guyonnet, V.; Hemsworth, P. H.; Huertas, S. M.; Huzzey, J. M.; Mellor, D. J.; Mench, J. A.; Spinka, M. and Whay, H. R. 2013. General principles for the welfare of animals in production systems: the underlying science and its application. The Veterinary Journal 198:19-27.

Gonyou, H. W.; Lemay, S. P. and Zhang Y. 2006. Effects of the environment on productivity and disease. p.1027-1038. In: Diseases of swine. 9th ed. Straw, B. E.; Zimmerman, J. J.; D'Allaire, S. and Taylor, D., eds. Blackwell Publishing, Ames, Iowa.

Hart, B. L. 1988. Biological basis of the behavior of sick animals. Neuroscience \& Biobehavioral Reviews 12:123-137.

Holyoake, T.; Richards, K. and McKenzie, P. 2012. Sick and injured pig guidelines for veterinarians. Available at: <www.ava.com. $\mathrm{au} / \mathrm{sites} / \mathrm{default} / \mathrm{files} / \mathrm{AVA}$ _website/pdfs/201203_APVGuidelines_ 1.7 noAd(interactive).pdf $>$. Accessed on: Aug. 10, 2016.

Ludtke, C. B.; Ciocca, J. R. P.; Dandin, T.; Barbalho, P. C.; Vilela, J. A. and Dalla Costa, O. A. 2010. Abate humanitário de suínos. WSPA, Rio de Janeiro.

Machado, I. P. 2014. Fluxo de produção e dimensionamento de instalações. p.106-110. In: Produção de suínos: Teoria e prática. Ferreira, A. H.; Carraro, B.; Dallanora, D.; Machado, G.; Machado, I. P.; Pinheiro, R. and Rohr, S. (Coord.). ABCS, Brasília.

Morés, N. 2006. Sala hospital para recuperação de suínos em uma granja.Available at: <https://www.embrapa.br/documents/1355242/ 1529323/Su\%C3\%ADnos+e+Aves+38-2006.pdf/48db7ca0-7b074db6-a67f-34e6b9430166>. Accessed on: Jul. 12, 2017.
Morés, N. 2007. Sala hospital e recuperação de suínos. p.120-124. In: Anais do 13ํㅡㄹ Congresso Brasileiro de Veterinários Especialistas em Suínos. Florianópolis, Brasil.

Mullan, S.; Edwards, S. A.; Butterworth, A.; Whay, H. R. and Main, D. C. J. 2009. Interdependence of welfare outcome measures and potential confounding factors on finishing pig farms. Applied Animal Behaviour Science 121:25-31.

Munsterhjelm, C.; Heinonen, M. and Valros, A. 2015. Effects of clinical lameness and tail biting lesions on voluntary feed intake in growing pigs. Livestock Science 181:210-219.

Otake, S.; Dee, S. A.; Rossow, K. D.; Deen, J.; Joo, H. S.; Molitor, T. W. and Pijoan, C. 2002. Transmission of porcine reproductive and respiratory syndrome virus by fomites (boots and coveralls). Journal of Swine Health and Production 10:59-65.

Pineiro, C.; Morales, J.; Dereu, A.; Wuyts, N.; Azlor, O.; Vizcaino, E. and Doncecchi, P. 2014. Individual Pig Care program improves productive performance and animal health in nursery-growing pigs. Journal of Swine Health and Production 22:296-299.

Rhim, S. J.; Son, S. H.; Hwang, H. S.; Lee, J. K. and Hong, J. K. 2015. Effects of mixing on the aggressive behavior of commercially housed pigs. Asian-Australasian Journal of Animal Sciences 28:1038-1043.

Stukenborg, A.; Traulsen, I.; Puppe, B.; Presuhn, U. and Krieter, J. 2011. Agonistic behaviour after mixing in pigs under commercial farm conditions. Applied Animal Behaviour Science 129:28-35.

Temple, D.; Courboulay, V.; Velarde, A.; Dalmau, A. and Manteca, X. 2012. The welfare of growing pigs in five different production systems in France and Spain: assessment of health. Animal Welfare 21:257-271

Temple, D.; Dalmau, A.; de la Torre, J. L. R.; Manteca, X. and Velarde, A. 2011. Application of the Welfare Quality ${ }^{\circledR}$ protocol to assess growing pigs kept under intensive conditions in Spain. Journal of Veterinary Behavior 6:138-149.

Temple, D.; Manteca, X.; Dalmau, A. and Velarde, A. 2013. Assessment of test-retest reliability of animal-based measures on growing pig farms. Livestock Science 151:35-45.

Thomsen, P. T.; Klottrup, A.; Steinmetz, H. and Herskin, M. S. 2016. Attitudes of Danish pig farmers towards requirements for hospital pens. Research in Veterinary Science 106:45-47.

van de Weerd, H. A. and Day, J. E. L. 2009. A review of environmental enrichment for pigs housed in intensive housing systems. Appied Animal Behaviour Science 116:1-20.

Weary, D. M.; Huzzey, J. M. and von Keyserlingk, M. A. G. 2009. Board-invited review: Using behavior to predict and identify ill health in animals. Journal of Animal Science 87:770-777.

Welfare Quality. 2009. Welfare quality assessment protocol for pigs (sows and piglets, growing and finishing pigs). Welfare Quality Consortium, Lelystad, the Netherlands.

Zulovich, J. M. 2012. Effect of the Environment on health. p.60-66. In: Diseases of Swine. 10th ed. Zimmerman, J. J.; Karriker, L. A.; Ramirez, A.; Schwartz, K. J. and Stevenson, G. W., eds. WileyBlackwell, John Wiley \& Sons, Inc., Publication, Ames, Iowa. 